\title{
A space-time finite element model for design and control optimization of nonlinear dynamic response
}

\author{
P.P. Moita ${ }^{\mathrm{a}}$, J.B. Cardoso ${ }^{\mathrm{b}, *}$ and A.J. Valido ${ }^{\mathrm{a}}$ \\ ${ }^{a}$ Escola Superior de Tecnologia de Setúbal, Campus do IPS, 2914-508 Setúbal, Portugal \\ ${ }^{\mathrm{b}}$ Instituto Superior Técnico, Departamento de Engenharia Mecânica, Av. Rovisco Pais, 1049-001 Lisboa, Portugal
}

Received 2007

Revised 2007

\begin{abstract}
A design and control sensitivity analysis and multicriteria optimization formulation is derived for flexible mechanical systems. This formulation is implemented in an optimum design code and it is applied to the nonlinear dynamic response. By extending the spatial domain to the space-time domain and treating the design variables as control variables that do not change with time, the design space is included in the control space. Thus, one can unify in one single formulation the problems of optimum design and optimal control. Structural dimensions as well as lumped damping and stiffness parameters plus control driven forces, are considered as decision variables. The dynamic response and its sensitivity with respect to the design and control variables are discretized via space-time finite elements, and are integrated at-once, as it is traditionally used for static response. The adjoint system approach is used to determine the design sensitivities. Design optimization numerical examples are performed. Nonlinear programming and optimality criteria may be used for the optimization process. A normalized weighted bound formulation is used to handle multicriteria problems.
\end{abstract}

Keywords: Optimization, nonlinear systems, dynamic response, space-time finite elements

\section{Introduction}

Structures and flexible mechanical systems, on the one hand, as well as optimal design and optimal control, on the other hand, have been traditionally treated with separated formulations. As the theory and methods of nonlinear structural analysis have progressed, there is no distinction between flexible mechanical systems and structures. Also, in the last decades there has been the integration of optimal design and optimal control problems [1-5].

This paper presents an integrated methodology for optimal design and control of nonlinear flexible mechanical systems. In order to implement it, one uses: (i) a nonlinear structural finite element technique to model large displacements, referring all the quantities to an inertial frame and using stress and strain measures that are invariant with the rigid body motion; (ii) a conceptual separation between time variant and time invariant design parameters, this way including the design space into the control space and considering the design variables as control variables not depending on time.

By using time integrals through all the derivations, the design and control problems are unified. In the optimization process we can use both types of variables simultaneously or by interdependent levels. Both types are designated here as design variables. A bound formulation is applied to handle the multicriteria problem. To model the systems,

${ }^{*}$ Corresponding author. E-mail: barradas @ist.utl.pt. 
a space-time finite element discretization is utilized and the solution of the dynamic equations is obtained by at-once integration.

The aim of the Design Sensitivity Analysis (DSA) is the gradients calculation of the performance measures with respect to the design variables. It represents an important tool for design improvement and it is a necessary stage within the optimization process. A general overview of the DSA problems and methods of nonlinear structural mechanics is given elsewhere [6]. Basically, the methods may be classified as direct differential methods and adjoint system methods. In this work one is going to use the adjoint method.

The response analysis and corresponding DSA are implemented in the interactive optimal design code OPTIMISE in order to use optimality criteria or nonlinear programming optimization runs.

\section{Response analysis modeling}

The virtual work dynamic equilibrium equation of the system at the time $t$ is given as

$$
\delta W=\int\left(\rho^{t} \ddot{u} \cdot \delta^{t} u+{ }^{t} S \cdot \delta^{t} \varepsilon-{ }^{t} f \cdot \delta^{t} u\right)^{0} d V-\int{ }^{t} T \cdot \delta^{t} u^{0} d \Gamma
$$

where all the quantities are referred to the undeformed configuration, $\delta$ represents variation of the state fields, '.' refers to the standard tensor product, upper dot ' '. refers to the material time derivative, $\rho$ is the mass density at time $t=0,{ }^{t} u$ is the displacement, ${ }^{t} S$ is the second-Piola stress measure, ${ }^{t} \varepsilon$ is the Green strain tensor, ${ }^{t} f$ is the body force per unit volume, ${ }^{t} T$ is the surface traction, ${ }^{0} V$ is the undeformed volume of the body, and ${ }^{0} \Gamma$ is the surface of the undeformed body.

A space-time finite element model is used to discretize the dynamic analysis response. After space discretization we have the governing matrix equation as

$$
M^{t} \ddot{U}+{ }^{t} C_{S}^{t} \dot{U}+{ }^{t} K_{S}^{t} U={ }^{t} P
$$

where $M$ is the mass matrix ${ }^{t} C_{S} \equiv{ }^{t} C_{S}\left({ }^{t} \dot{U}\right),{ }^{t} K_{S} \equiv{ }^{t} K_{S}\left({ }^{t} U\right)$ are respectively the damping and stiffness matrices, ${ }^{t} P$ is the loading vector and ${ }^{t} U,{ }^{t} \dot{U},{ }^{t} \ddot{U}$ are respectively the displacement, velocity and acceleration vectors, all the quantities defined at time $t$.

For temporal modeling, the algorithm given in [7] is extended to the case of nonlinear systems. Finite elements of dimension $\Delta t$ were considered, selecting hermitean cubic elements to model the displacements and quadratic lagrangean elements to model the loading. By taking the time derivative of the Eq. (2) on the one hand, and on the other hand integrating it once and then twice with average values of stiffness and damping in $\Delta t$ given as

$$
\bar{K} \equiv{ }^{t+\Delta t / 2} K_{S}\left({ }^{t} U+{ }^{t} \dot{U} \Delta t / 2\right), \bar{C} \equiv{ }^{t+\Delta t / 2} C_{S}\left({ }^{t} \dot{U}+{ }^{t} \ddot{U} \Delta t / 2\right)
$$

one obtains four equations that combine to give the dynamic finite time-element equation as

$$
D_{S}^{e} z^{e}=P^{e}
$$

where

$$
D_{S}^{e}=\left[\begin{array}{llllll}
{ }^{t} K_{S} & { }^{t} C_{S} & M & 0_{n x n} & 0_{n x n} & 0_{n x n} \\
{ }^{t} D_{S_{11}}{ }^{t} D_{S_{12}} & 0_{n x n}{ }^{t+\Delta t} D_{S_{11}}{ }^{t+\Delta t} D_{S_{12}} & 0_{n x n} \\
{ }^{t} D_{S_{21}}{ }^{t} D_{S_{22}} & 0_{n x n} & { }^{t+\Delta t} D_{S_{21}}{ }^{t+\Delta t} D_{S_{22}} & 0_{n x n}
\end{array}\right], \quad P^{e}=\left\{\begin{array}{l}
{ }^{t} P \\
P_{1} \\
P_{2}
\end{array}\right\}
$$

and

$$
z^{e}=\left({ }^{t} z,{ }^{t+\Delta t} z\right), \quad{ }^{t} z=\left({ }^{t} U,{ }^{t} \dot{U},{ }^{t} \ddot{U}\right)
$$

In Eq. (5) $n$ is the number of space degrees-of-freedom and submatrices ${ }^{t} D_{S_{j k}}$ are functions of $\bar{K}, \bar{C}, M$. The Eq. (4) may be solved step-by-step, i.e., element-by-element in time, or assembled to be solved at-once. In this case, we have to assemble for a total time interval $T$ discretized in $\mathrm{N}$ time nodes, resulting in the dynamic equation

$$
D_{S} z=P
$$

Now, $2 n$ time boundary conditions are imposed by transferring the corresponding columns of the assembled matrix $D_{S}$ to the right-hand side of Eq. (7) after multiplying them by the vector $U_{c}$ of those conditions, resulting the equation

$$
\hat{K}_{S} \hat{U}=\hat{P}, \quad \hat{P}=P-D_{c} U_{c}
$$

The Eq. (8) is a nonlinear equation where $\hat{K}_{S}$ is a nonsymmetrical dynamic stiffness matrix dependent on the response. Therefore, the Eq. (8) has to be solved iteratively. 


\section{Design sensitivity analysis modeling}

Consider now a general performance measure defined in the time interval $[0, \mathrm{~T}]$ as

$$
\Psi=\int G\left({ }^{t} S,{ }^{t} \varepsilon,{ }^{t} z,{ }^{t} b, t\right) d t
$$

where ${ }^{t} b$ is the vector of design and control variables (let us call it "design vector") and the other quantities were defined in Eqs (1) and (6). The forces ${ }^{t} P$ belong in general to the design vector. The design sensitivity analysis problem is to derive the total design variation of the measure of the Eq. (9) with respect to the design ${ }^{t} b$, for the system represented by the equation of motion, Eq. (8).

The total design variation for the performance measure of Eq. (9) is

$$
\bar{\delta} \Psi=\overline{\bar{\delta}} \Psi+\tilde{\delta} \Psi
$$

where $\overline{\bar{\delta}} \Psi$ and $\tilde{\delta} \Psi$ represent respectively the explicit and implicit (state dependent) design variations. In order to formulate design sensitivity analysis, the adjoint system method is used. Therefore, replace the arbitrary variation $\delta \widehat{U}$ of state fields by adjoint fields $\hat{U}^{a}$ into the discretized virtual work equation as

$$
W^{a}=\left(\hat{K}_{S} \hat{U}-\hat{P}\right) \cdot \hat{U}^{a}=0
$$

and define an extended 'action' function

$$
A=\Psi-W^{a}
$$

The basic idea of introducing an adjoint system is to replace the implicit design variations of the state fields by explicit design variations and adjoint fields, then determining these adjoint fields by vanishing the implicit design variation of the 'action' function $A[4]$ as

$$
\tilde{\delta} A=0
$$

then the total design variation of the functional $\Psi$ can be written as

$$
\bar{\delta} \Psi=\overline{\bar{\delta}} A
$$

In order to solve the design sensitivity analysis problem, the sensitivities are firstly performed at the element level and then the sensitivity equations are assembled. The explicit design variation of the vector of element total forces of Eq. (4) is

$$
\overline{\bar{\delta}} R^{e}=\overline{\bar{\delta}} P^{e}-\overline{\bar{\delta}} F^{e}, \quad F^{e}=D_{S}^{e} z^{e}
$$

and the implicit design variation of the internal forces gives

$$
\tilde{\delta} F^{e}=\tilde{\delta} D_{S}^{e} z^{e}+D_{S}^{e} \tilde{\delta} z^{e}=D^{e} \tilde{\delta} z^{e}
$$

where

$$
D^{e}=\left[\begin{array}{llllll}
{ }^{t} K & { }^{t} C & M & 0_{n x n} & 0_{n x n} & 0_{n x n} \\
{ }^{t} D_{11}{ }^{t} D_{12} & { }^{t} D_{13} & { }^{t+\Delta t} D_{11}{ }^{t+\Delta t} D_{12} & 0_{n x n} \\
{ }^{t} D_{21} & { }^{t} D_{22} & { }^{t} D_{23} & { }^{t+\Delta t} D_{21} & { }^{t+\Delta t} D_{22} & 0_{n x n}
\end{array}\right]
$$

with

$$
{ }^{t} K={ }^{t} K_{S}+{ }^{t} K_{S, U}{ }^{t} U, \quad{ }^{t} C={ }^{t} C_{S}+{ }^{t} C_{S}, \dot{U}{ }^{t} \dot{U}
$$

Sensitivities of Eq. (15) and the element dynamic matrix of Eq. (17) are assembled and again imposed the time boundary conditions resulting respectively $\overline{\bar{\delta}} \hat{R}$ and $\hat{K}$.

Now, the adjoint system equilibrium of the Eq. (13) is

$$
\hat{K}^{T} \hat{U}^{a}=(\Psi, \hat{U})^{T}
$$

and the total design variation of Eq. (14) is given as

$$
\bar{\delta} \Psi=\overline{\bar{\delta}} \Psi+\hat{U}^{a} \cdot \overline{\bar{\delta}} \hat{R}
$$




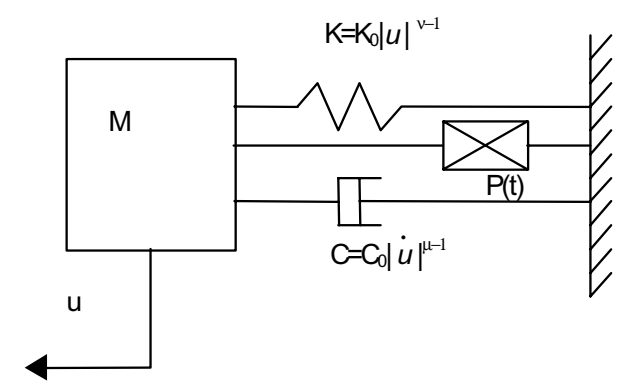

Fig. 1. Impact absorber.

\section{The optimum design problem}

The optimum design problem is to find the set of design variables ${ }^{t} b$ plus a new design variable $\beta$ in order to

$$
\begin{array}{ll} 
& \min \beta \\
\text { s.t. } & \Psi_{0 i} \leqslant \beta ; \quad i=1,2, \ldots m_{0} \\
& \Psi_{j} \leqslant 0 ; \quad j=1,2, \ldots m \\
& b_{i l} \leqslant b_{i} \leqslant b_{i u} ; \quad i=1,2, \ldots, n+1 ; \quad b_{n+1} \equiv \beta
\end{array}
$$

where $\Psi_{0 i}$ are the original objectives, $\Psi_{j}$ are the original constraints and $b_{i l}, b_{i u}$ stand for the lower and upper bounds of the design variables. The formulation of the Eq. (21) is a bound formulation where the new variable $\beta$ sets an upper bound to the original objectives, then its minimization corresponds to a multi-objective minimization [9]. Particularly, it may correspond to a one-objective minimization.

In order to handle different objectives, these are in the Eq. (21) normalized accordingly to

$$
\Psi_{0 i}=\left(\bar{\Psi}_{0 i}-\bar{\Psi}_{0 i}^{\min }\right) w_{i} /\left(\bar{\Psi}_{0 i}^{\max }-\bar{\Psi}_{0 i}\right), \quad \sum w_{i}=1
$$

In the Eq. (22), $\bar{\Psi}_{0 i}$ is the $\mathrm{i}^{\text {th }}$ original objective, $w_{i}$ represents a weight given by the designer accordingly to his or her preference, $\bar{\Psi}_{0 i}^{\min }$ is its single objective attainable minimum and $\bar{\Psi}_{0 i}^{\max }$ is its maximum value obtained value when all the other single objectives minimizations are performed.

\section{Examples}

\subsection{Nonlinear impact absorber}

The system of the Fig. 1 may represent a landing gear for an aircraft impacting the ground at a certain velocity $v=1 \mathrm{~m} / \mathrm{s}$ at $t=0$. The problem is to find the spring and damping coefficients $K_{0}|u|^{\nu-1}$ and $C_{0}|\dot{u}|^{\mu-1}$, and the control force $[P(t), 0 \leqslant t \leqslant 12 s]$, satisfying a displacement constraint $|u| \leqslant 1$ with $\nu=\mu=2$, such that: (I) minimizes the maximum acceleration $|a|$ of the mass $M=1 \mathrm{~kg}$, with starting decision variables at $K_{0}=0.5 \mathrm{~N} / \mathrm{m}$, $C_{0}=0.5 \mathrm{Ns} / \mathrm{m}$ and $P(t)=0$; (II) minimizes the bicriteria $\left\{\Psi_{01} \equiv C_{0}, \Psi_{02} \equiv E=\int\left(100 \ddot{u}^{2}+\dot{u}^{2}+100 P^{2}\right) d t\right\}$ with starting decision variables at $K_{0}=0.597 \mathrm{~N} / \mathrm{m}, C_{0}=0.597 \mathrm{Ns} / \mathrm{m}$ and $P(t)=0$.

In the Case I, the optimum design was obtained by sequential quadratic programming as $K_{0}=0.00667 \mathrm{~N} / \mathrm{m}$, $C_{0}=6.96 \mathrm{Ns} / \mathrm{m}$. Starting and optimal acceleration responses are obtained for different levels of the optimization process (Fig. 2). The optimal control $P(t)$ is shown in Fig. 4. The responses were obtained by at-once integration.

In the Case II, a normalized weighted bound formulation was used to handle the bicriteria problem. The normalization of the objectives was made according to the Eq. (22).

The bound formulation may be written as to find the control $P(t)$, the original design variables $K_{0}, C_{0}$, and a new design variable $\beta$, bounding the objectives, that is

$$
\min \beta \text {, s.t. } \Psi_{0 i} \leqslant \beta, \quad|u| \leqslant 1
$$




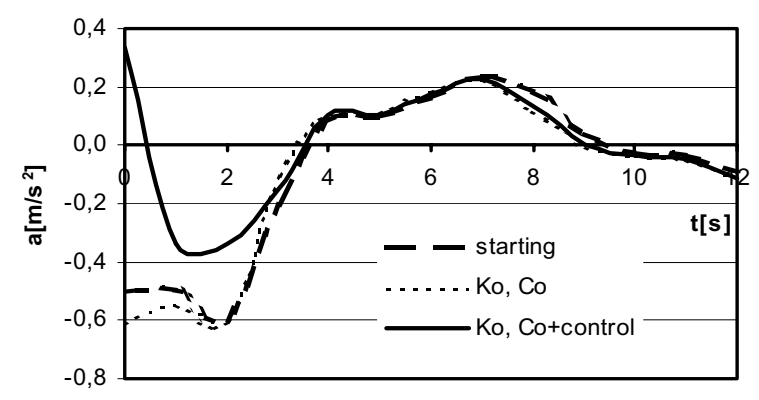

Fig. 2. Impact absorber max. acceleration (Case I).

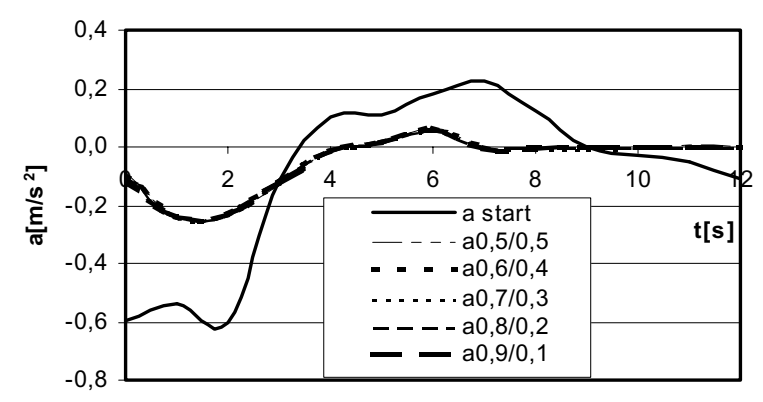

Fig. 3. Impact absorber max. acceleration (Case II).

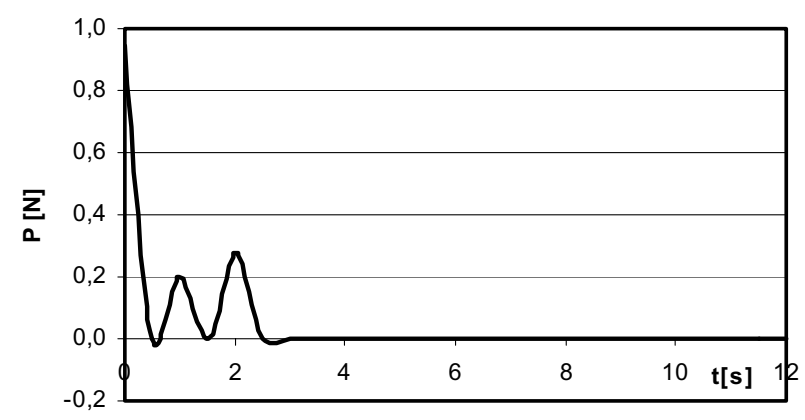

Fig. 4. Optimal control for impact absorber (Case I).

The optimum design was obtained by sequential quadratic programming and various runs were made using various weight combinations of the objectives $C_{0}$ and $E$, which are given in the Table 1.

The optimum maximum acceleration responses are shown in the Fig. 3. The optimum design variables are given in the Table 1 and the optimum control driven forces are represented in the Fig. 5. The criteria space is represented in Fig. 6. The almost linearity of the curve of the optima suggests we cannot find a convenient point where we can trade off the weight of one objective having a small increase in the other objective.

\subsection{Vehicle suspension}

A ground obstacle excites kinematically a vehicle suspension (Fig. 7). The tires are represented by springs $k 4=k 5=26.275 \mathrm{~N} / \mathrm{m}$ and by shock absorbers $c 4=c 5=875.8 \mathrm{Ns} / \mathrm{m}$. The chassis body is partially discretized by beam elements with specific mass $\rho=7800 \mathrm{~kg} / \mathrm{m}^{3}$ and Young modulus $E=200 \mathrm{GPa}$ and partially as concentrated masses. The ratio 2nd moment of area/chassis cross-section area of $I / A=0.01 \mathrm{~m}^{2}$ is considered. Other masses are the mass of the driver $m 1=131.6 \mathrm{~kg}$, and the mass of the wheels $m 3=m 4=43.6 \mathrm{~kg}$. The distance between front and rear wheels is $3.048 \mathrm{~m}$ and the vehicle moves at $87.78 \mathrm{Km} / \mathrm{h}$. 
Table 1

Optimal $K_{0}$ and $C_{0}$ with various objective weights

\begin{tabular}{ccc}
\hline $\mathrm{w}_{E} / \mathrm{w}_{C o}$ & $K_{\text {Ooptimum }}$ & $C_{\text {Ooptimum }}$ \\
\hline $0.5 / 0.5$ & 0.458 & 0.015 \\
$0.6 / 0.4$ & 0.453 & 0.022 \\
$0.7 / 0.3$ & 0.450 & 0.032 \\
$0.8 / 0.2$ & 0.446 & 0.050 \\
$0.9 / 0.1$ & 0.442 & 0.091 \\
\hline
\end{tabular}

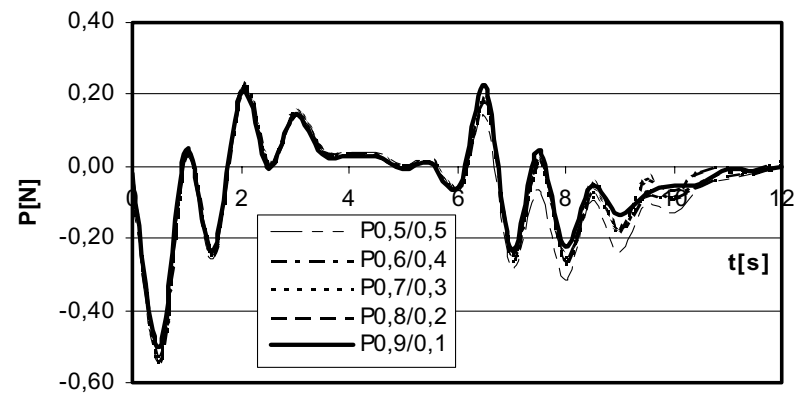

Fig. 5. Optimal control for impact absorber (Case II).

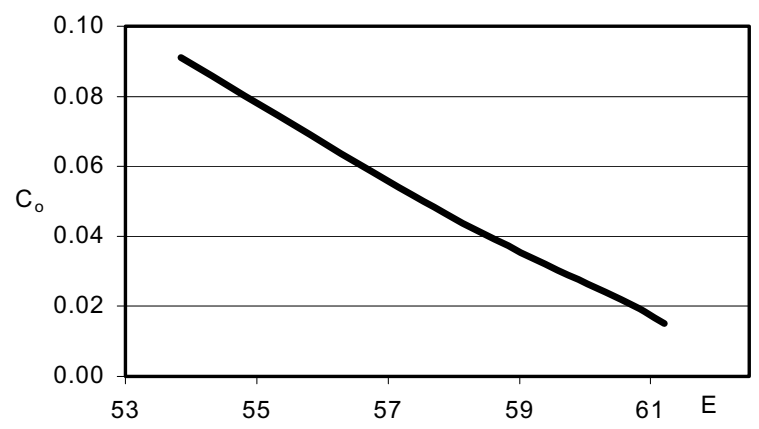

Fig. 6. Criterium space.

The optimization problem is to minimize the maximum acceleration magnitude of the mass $m 1$ such that the relative vertical displacement between masses is not larger than $0.05 \mathrm{~m}$ for a ground sinusoidal obstacle of height $0.1016 \mathrm{~m}$ and wavelength $24.384 \mathrm{~m}$. Starting design is $\left\{A=0.1 \mathrm{~m}^{2}, k 1=1.75 \mathrm{E} 4, k 2=k 3=5.26 \mathrm{E} 4 \mathrm{~N} / \mathrm{m}, c 1=\right.$ $1.75 \mathrm{E} 3, c 2=c 3=4.38 \mathrm{E} 3 \mathrm{Ns} / \mathrm{m}\}$, and starting control is $[P(t)=0 ., 0 \leqslant t \leqslant 1 s]$, where $P$ is the actuator control force in the passenger seat. Sequential quadratic programming was used for the optimization process.

The optimal design was obtained as $\{0.35579,6.94 \mathrm{E} 4,6.31 \mathrm{E} 4,5.02 \mathrm{E} 4,1 . \mathrm{E} 6,1.34 \mathrm{E} 4,1 . \mathrm{E} 2\}$ and the optimal control is represented in Fig. 8. Starting and optimal acceleration responses are shown in Fig. 9, for different levels of the optimization process.

\section{Concluding remarks}

By extending the spatial domain to the space-time domain, the design space is included in the control space. Thus, one can unify in one single formulation the problems of optimum design and optimal control, treating the design variables as control variables that do not change with time.

Using at-once integration of the equations of motion and its sensitivities, the adjoint method of design sensitivity analysis can be used with all of its advantages and without its main disadvantage in dynamics: the necessity of backwards integration of the adjoint system. So, the needing of backwards integration with its major drawback - the 


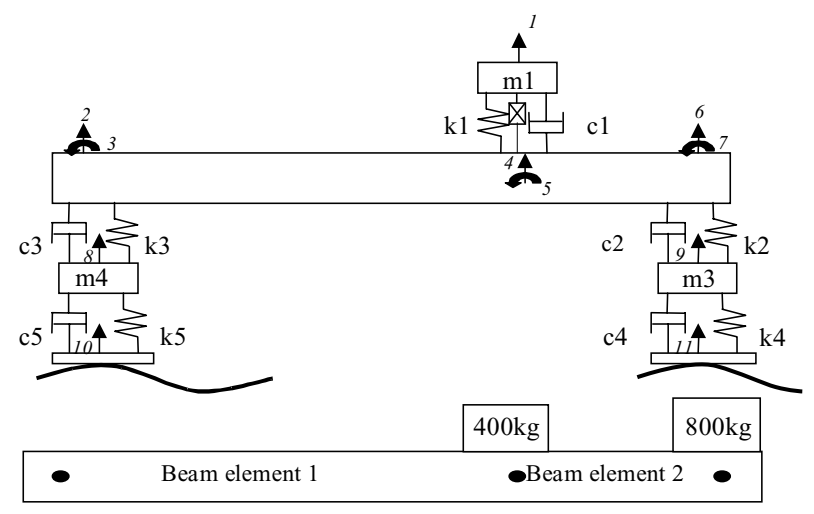

Fig. 7. Vehicle suspension.

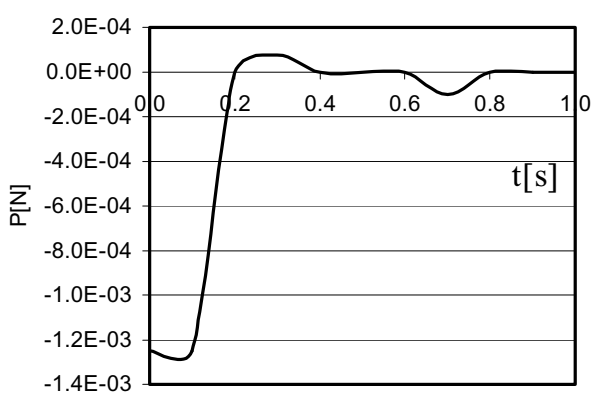

Fig. 8. Vehicle suspension optimal control $[\mathrm{N}]$.

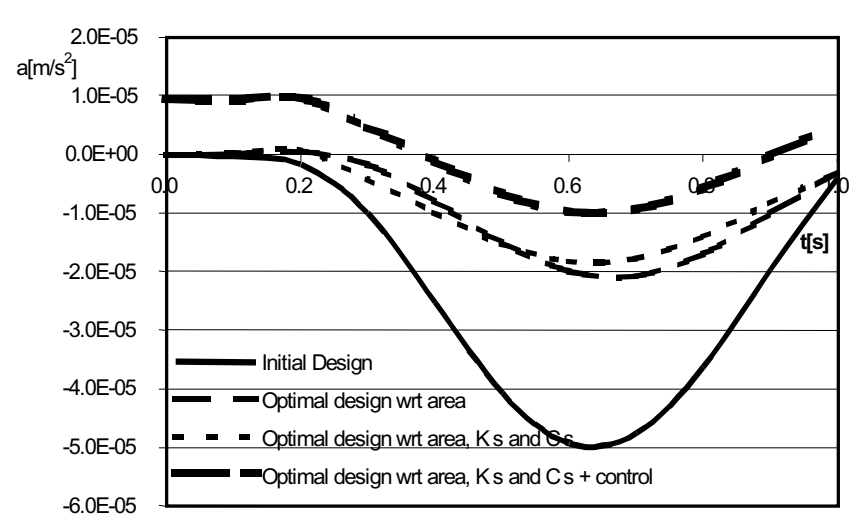

Fig. 9. Vehicle suspension max. acceleration $\left[\mathrm{m} / \mathrm{s}^{2}\right]$.

memorization of the response history - is not associated with path-dependent problems, as it is frequently assumed, but it is associated with the fact of using step-by-step integration.

It is also possible and easy to apply temporal boundary conditions at any point in time, not necessarily at the start of the integration, as it is usual for the step-by-step integration method.

\section{References}

[1] M. Salama, J. Garba, L. Demsetz and F. Udwadia, Simultaneous Optimization of Controlled Structures, Computational Mechanics Mech 3 (1988), 275-282. 
[2] C.H. Tseng and J.S. Arora, Optimum Design of Systems for Dynamics and Controls using Sequential Quadratic Programming, AIAA Journal 27(12) (1989), 1793-1800.

[3] J.S. Arora and T.C. Lin, A Study of Augmented Lagrangean Methods for Simultaneous Optimization of Controls and Structures, Fourth AIAA/Air Force/NASA/OAI Symposium, Cleveland, Ohio, USA, 1992.

[4] J.B. Cardoso and J.S. Arora, Design Sensitivity Analysis of Nonlinear Dynamic Response of Structural and Mechanical Systems, Structural Optimization 4 (1992), 37-46.

[5] J.B. Cardoso, L. Sousa, J. Castro and A. Valido, Optimal Design of Nonlinear Structures and Mechanical Systems, Engineering Optimization 29 (1997), 277-291.

[6] M. Kleiber, H. Antunez, T. Hien and P. Kowalczyk, Parameter Sensitivity in Nonlinear Mechanics, John Wiley \& Sons, 1997.

[7] M. Gellert, A New Algorithm for Integration of Dynamic Systems, Computers \& Structures 9 (1978), 401-408.

[8] J.S. Arora, Introduction to Optimum Design, Elsevier Academic Press, 2005.

[9] R.T. Marler and J.S. Arora, Survey of Multi-Objective Optimization Methods for Engineering, Structural and Multidisciplinary Optimization 26 (2004), 369-395. 

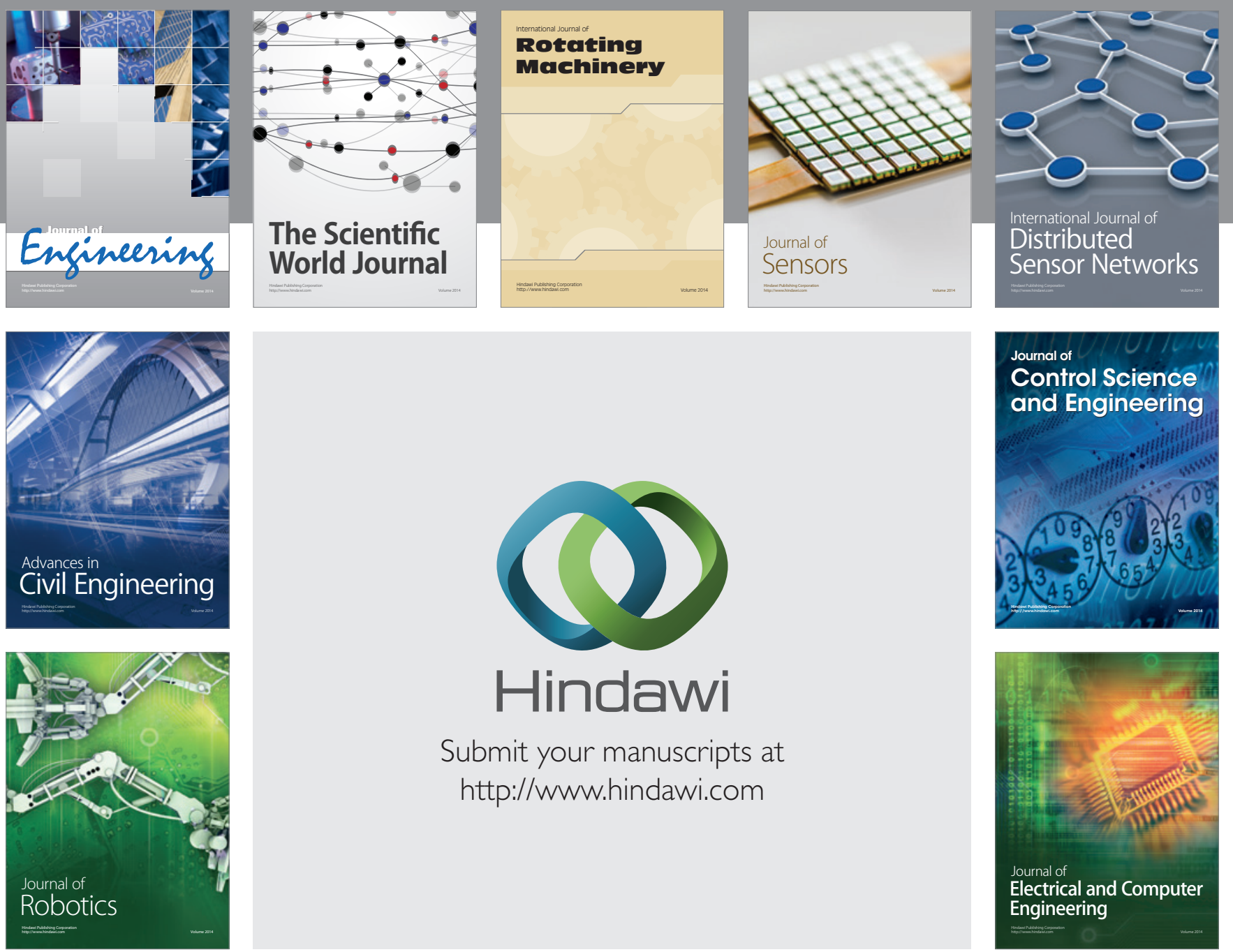

Submit your manuscripts at

http://www.hindawi.com
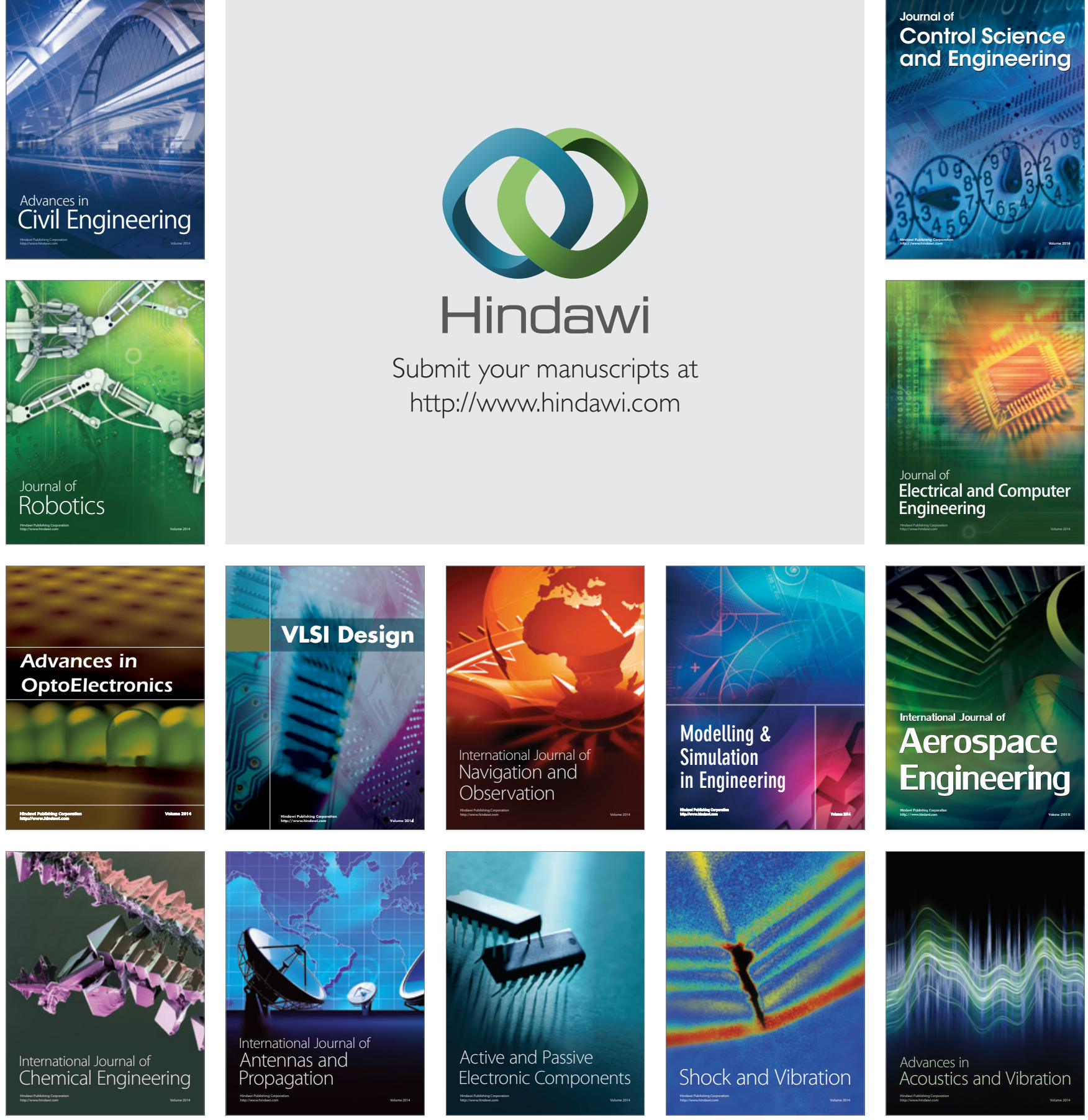